\title{
Talaromyces marneffei fungemia after travel to China in a Canadian patient with AIDS
}

\author{
Mara Waters MD, Alina Beliavsky MD, Kevin Gough MD MEd
}

Cite as: CMAJ 2020 January 27;192:E92-5. doi: 10.1503/cmaj.191136

A 62-year-old man was admitted to the general medicine service with a 2-month history of odynophagia, fevers, rigors, a 22-kg unintentional weight loss and watery diarrhea. He had moved to Canada 25 years earlier from Guangzhou, China (Guangdong Province) and had last visited China 1 year before presentation.

The patient had been diagnosed with HIV infection 20 years earlier. One year before presentation, his CD4 count and viral load were 24 cells $/ \mu \mathrm{L}$ and 144 copies/mL, respectively. He had been treated for Pneumocystis jirovecii pneumonia on 2 occasions, 12 and 20 years previously. He intermittently complied with his antiretroviral therapy (emtricitabine, tenofovir, darunavir, ritonavir and raltegravir) and his opportunistic infection prophylaxis (azithromycin for Mycobacterium avium complex prophylaxis, and trimethoprim-sulfamethoxazole for $P$. jirovecii pneumonia and toxoplasmosis prophylaxis). Other medical comorbidities included hepatitis $B$ coinfection and chronic obstructive pulmonary disease.

On initial examination, the patient appeared thin and cachectic. Examination of the heart and lungs was within normal limits. There was no hepatosplenomegaly or palpable lymphadenopathy. The oropharynx was clear. Skin examination showed red papular lesions scattered on the patient's face and back. He had daily fever (temperature $>38^{\circ} \mathrm{C}$ ) for the first week of his hospital stay, with a maximum temperature of $38.4^{\circ} \mathrm{C}$. At presentation, his absolute CD4 count was 0 cells $/ \mu \mathrm{L}$, and his viral load was 3243 copies $/ \mathrm{mL}$. He had severe neutropenia (nadir $0.2 \times 10^{9} / \mathrm{L}$ ) and thrombocytopenia (nadir $32 \times 10^{9} / \mathrm{L}$ ). His liver enzyme levels were mildly elevated (aspartate aminotransferase $88 \mathrm{U} / \mathrm{L}$, alanine aminotransferase $39 \mathrm{U} / \mathrm{L}$ and alkaline phosphatase $128 \mathrm{IU} / \mathrm{L}$ ). His renal function and electrolytes were within normal limits.

On hospital day 5, a filamentous fungus was isolated in 3 peripheral blood culture bottles after 5 days of incubation in an automatic incubator. Subsequent speciation identified Talaromyces marneffei (Figure 1). Stool examination for ova and parasites identified Clonorchis sinensis eggs. Stool culture was negative for Campylobacter, Escherichia coli 0157, Salmonella, Yersinia and Shigella. Multiple sputum samples were negative for acidfast bacilli. Serologic testing gave negative results for hepatitis $C$ antibody and Strongyloides stercoralis IgG antibody by enzymelinked immunosorbent assay, and positive results for hepatitis B

\section{KEY POINTS}

- Talaromyces marneffei should be considered in immunocompromised people with a travel history to endemic areas including southeast Asia, northern India and southern China.

- Characteristic presentation features may include several weeks of fevers, weight loss and skin lesions (papules, ulcers or abscesses).

- Diagnosis is made by fungal culture and confirmed through molecular techniques such as the polymerase chain reaction assay; however, diagnosis may be delayed if laboratories are not equipped with these techniques or samples need to be sent to reference laboratories.

- Treatment of T. marneffei fungemia is with liposomal amphotericin B induction for 2 weeks, followed by oral itraconazole therapy for 10 weeks.

- Primary prophylaxis with oral itraconazole therapy should be considered for patients with HIV infection and CD4 counts less than 100 cells/ $\mu \mathrm{L}$ who reside in or travel to endemic areas.

surface antigen, e antigen and core antibody. Biopsy of a skin lesion showed nonspecific interface dermatitis, with no fungal elements seen on periodic acid-Schiff staining; fungal cultures of the skin lesion were not performed. Gastroscopy showed mild gastritis, and Helicobacter pylori was identified on histopathological examination. Computed tomography of the thorax, abdomen, pelvis and head showed retroperitoneal and mesenteric lymphadenopathy, with no findings suggestive of metastatic fungal disease. Ultrasonography of the abdomen showed mild hepatic steatosis; the gallbladder was normal.

The infectious diseases service was consulted, and treatment of the T. marneffei fungemia was initiated with liposomal amphotericin B, $4 \mathrm{mg} / \mathrm{kg}$ daily for a 2-week induction period, followed by treatment-dose itraconazole, $200 \mathrm{mg}$ administered orally twice daily for 10 weeks. The patient's fevers, odynophagia and diarrheal symptoms resolved over 2 weeks. The $C$. sinensis parasitemia was treated with praziquantel, $25 \mathrm{mg} / \mathrm{kg} 3$ times daily for 2 days. Helicobacter pylori treatment was advised, but the patient declined. His antiretroviral regimen was changed to emtricitabine, tenofovir, abacavir and dolutegravir to reduce drug interactions with his antifungal regimen. 


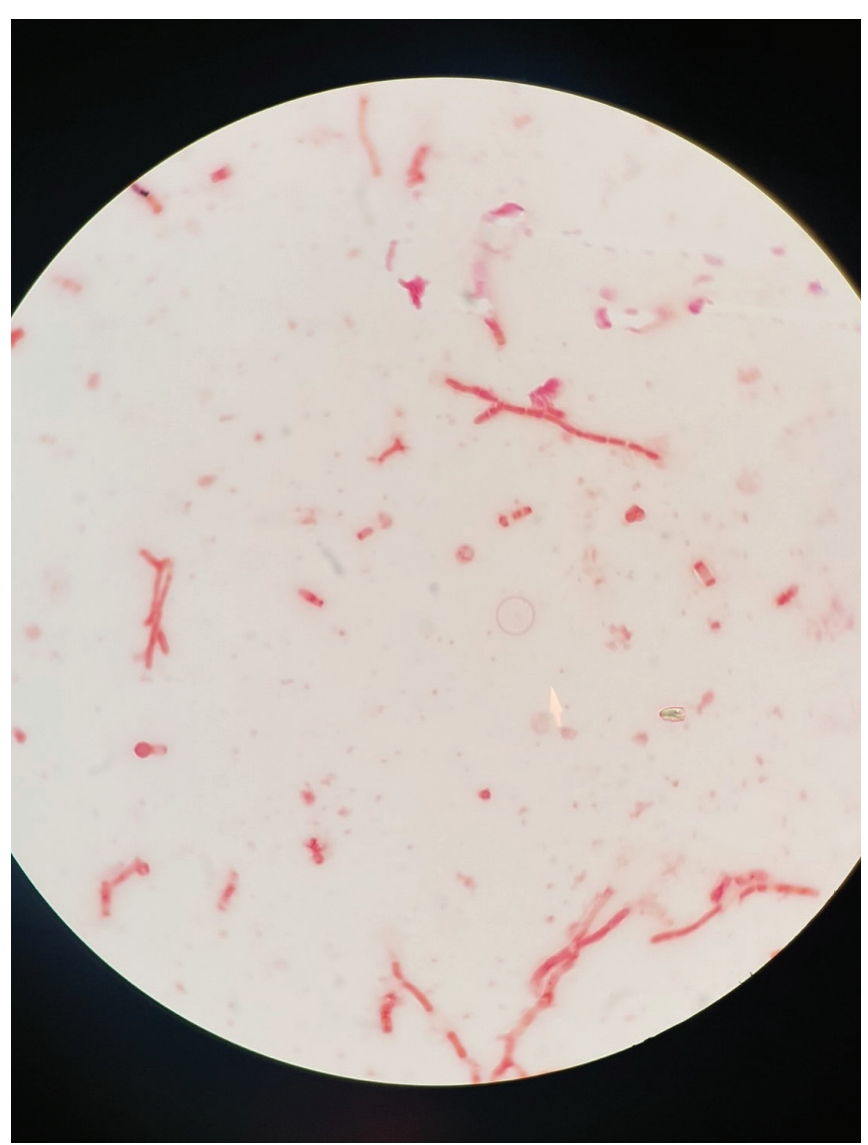

Figure 1: Talaromyces marneffei identified on a potassium hydroxide smear from a blood culture specimen. Yeast hyphae and arthroconidia were seen under light microscopy at $37^{\circ} \mathrm{C}$.

The patient was seen by the hematology service, who recommended granulocyte colony-stimulating factor for 5 days for his neutropenia. His thrombocytopenia may have been related to HIV-associated idiopathic thrombocytopenia purpura, the trimethoprim-sulfamethoxazole or the T. marneffei infection. At 4 months follow-up, he had a platelet count of $232 \times 10^{9} / \mathrm{L}$, neutrophil level of $0.5 \times 10^{9} / \mathrm{L}, \mathrm{CD} 4$ count of 7 cells $/ \mu \mathrm{L}$ and viral load of 451 copies $/ \mathrm{mL}$.

\section{Discussion}

Talaromyces marneffei is a dimorphic fungus endemic to southeast Asia, northern India and southern China (Figure 2). The overt disease incidence increased substantially with the rise of HIV infection in the 1980s, with a decline in incidence since effective antiretroviral therapy became widely instituted in the region. ${ }^{2}$ The main risk factor for T. marneffei disease is dysfunctional cellmediated immunity, commonly secondary to HIV infection, especially in people with a CD4 count less than 100 cells/ $\mu \mathrm{L}$. The incidence has been increasing among patients with immunodeficiency disorders involving the interleukin-12/interferon- $\gamma$ signalling pathway and among those receiving immunosuppressive T-cell-depleting agents (such as tacrolimus) or monoclonal antibodies (such as rituximab). These patients have higher mortality rates, potentially owing to lack of clinical suspicion. ${ }^{3}$
The bamboo rat is the only known nonhuman host of T. marneffei. ${ }^{4}$ However, there is little evidence of direct rat-tohuman transmission. Although the mode of transmission of T. marneffei remains unknown, exposure is likely environmental, with inhalation of $T$. marneffei conidia from environments such as soil or plants where bamboo rat feces may be present. ${ }^{4}$

The incubation period for T. marneffei is $1-3$ weeks in patients with acute disease. In addition, latent $T$. marneffei infection can occur, with disease reactivation at any time in immunocompromised hosts. ${ }^{5}$ Our patient's illness likely represented reactivation of latent disease in the context of a low CD4 count. He may have acquired the infection during his time of residence in Guangzhou or during one of his return trips, most recently 1 year before presentation at our institution.

\section{Disseminated Talaromyces marneffei infection}

Clinical features of disseminated $T$. marneffei infection may include fever, lymphadenopathy, weight loss, hepatomegaly, splenomegaly, respiratory and gastrointestinal abnormalities, and skin lesions. ${ }^{2,6}$ The skin lesions are present in $70 \%$ of cases and are classically described as necrotic papules, although they may vary in appearance. ${ }^{2}$ Because the other symptoms are nonspecific, the skin lesions may play an important role in prompting consideration of this diagnosis, increasing pretest probability and making the diagnosis through isolation of $T$. marneffei on biopsy. ${ }^{2,6}$ In a retrospective cohort study of patients with HIV/ AIDS in southern China, mortality was significantly greater in patients with $T$. marneffei than in those without, with an inhospital mortality rate of $17.5 \%$ and $7.6 \%$, respectively. ${ }^{7}$

Common laboratory findings in disseminated $T$. marneffei infection include elevated liver enzyme levels, anemia and thrombocytopenia. ${ }^{2,6}$

\section{Diagnosis}

Growing T. marneffei on culture is the gold standard method of diagnosis, followed by confirmation that involves showing mould to yeast (Figure 1) conversion, at $25^{\circ} \mathrm{C}$ and $37^{\circ} \mathrm{C}$ respectively. More recently, molecular techniques such as the polymerase chain reaction assay and enzyme-linked immunosorbent assay, which often must be performed at a reference laboratory, have been used in an effort to expedite the diagnosis. ${ }^{8}$ In our patient, both culture and the polymerase chain reaction assay were performed and yielded concordant positive results.

\section{Treatment}

Recommended treatment for severe disease with central nervous system involvement includes liposomal amphotericin B, $5 \mathrm{mg} / \mathrm{kg}$ for 4-6 weeks, followed by itraconazole, $400 \mathrm{mg} / \mathrm{d}$ given orally for 10 weeks, then secondary prophylaxis. ${ }^{9}$ Treatment for moderate and severe disease (multiorgan involvement with or without respiratory failure or shock) without central nervous system involvement includes induction with liposomal amphotericin B, 3-5 mg/kg daily for 2 weeks, followed by itraconazole, $400 \mathrm{mg} / \mathrm{d}$ given orally for 10 weeks; in the setting of HIV infection, maintenance therapy with itraconazole, $200 \mathrm{mg} / \mathrm{d}$ administered orally, should be given for secondary prophylaxis until the CD4 


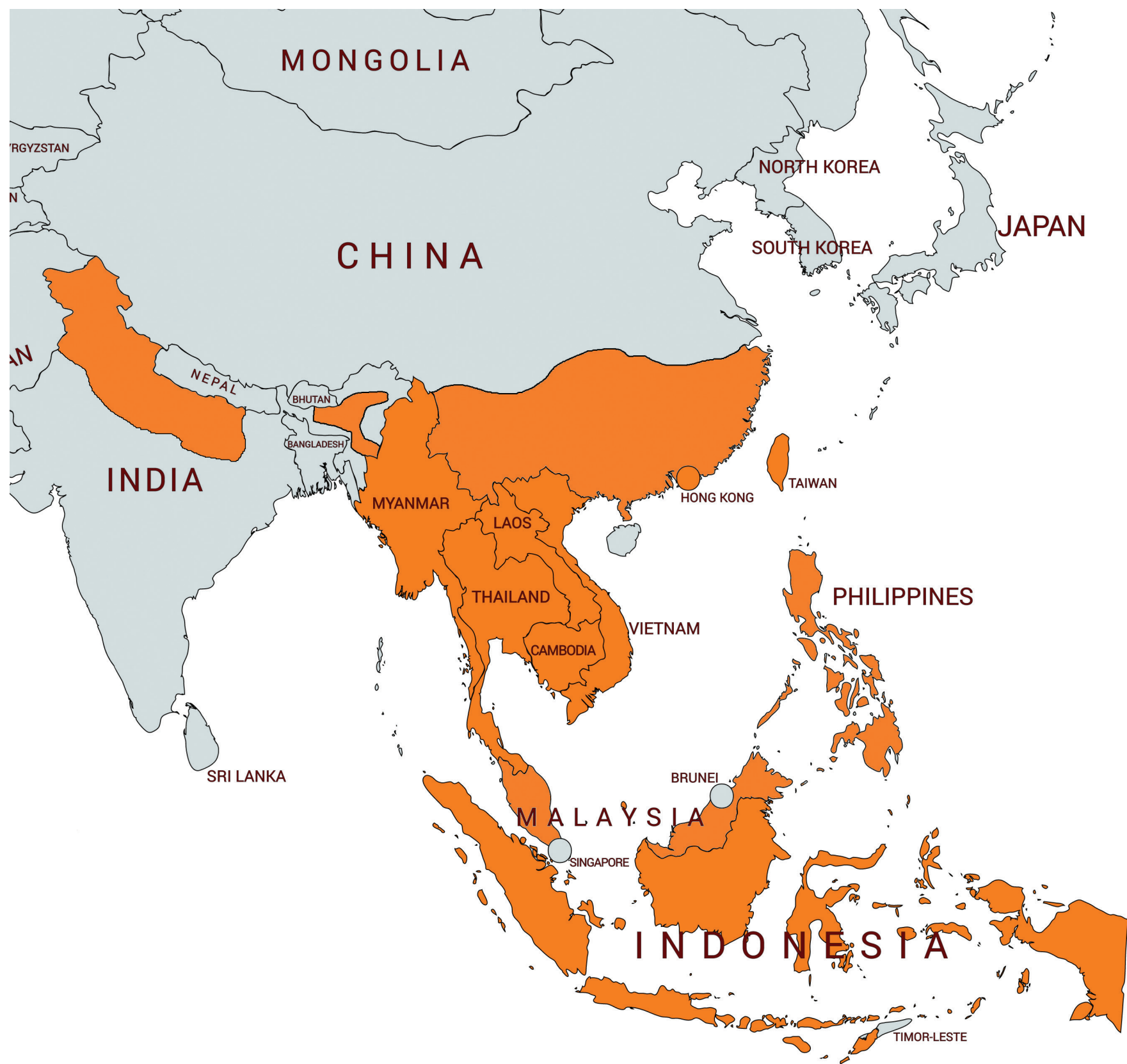

Figure 2: Map showing regions endemic for Talaromyces marneffei (orange shading). Adapted from reference 1 with permission from Leading International Fungal Infection (LIFE).

count is greater than 100 cells $/ \mu \mathrm{L} .{ }^{10}$ Mild disease (cutaneous involvement alone) may be treated with itraconazole, $400 \mathrm{mg} / \mathrm{d}$ for 8 weeks, followed by secondary prophylaxis. Itraconazole is a cytochrome P450 3A4 inhibitor; thus, prescribers must be cognizant of potential drug interactions.

\section{Primary prophylaxis}

Primary prophylaxis with itraconazole, $200 \mathrm{mg} / \mathrm{d}$ given orally, is indicated for patients with HIV infection and CD4 counts less than 100 cells/ $\mu \mathrm{L}$ who reside in or travel to areas endemic for T. marneffei in southeast Asia. ${ }^{10}$ Other infections in HIV-infected people may warrant primary prophylaxis (Box 1). Despite the risk of talaromycosis in patients without HIV infection who have immunodeficiency disorders or who take immunosuppressive medications, there are no current guidelines recommending primary prevention in these patients.

\section{Conclusion}

Our patient's presentation would be considered fairly common in parts of southeast Asia; however, it is uncommon in North America. ${ }^{11}$ This case highlights the importance of increasing awareness of T. marneffei in immunocompromised hosts given our diverse patient populations, the increasing use of immunosuppressive therapies and growing international travel. Establishing a correct diagnosis in a timely manner is important since effective treatment is available and manifestations and sequelae of the disease can be severe. 
Box 1: Primary prophylaxis of opportunistic infections with antimicrobial therapy in patients with HIV infection ${ }^{10}$

\section{Opportunistic}

infection

\section{CD4 count,} cells/ $\mu \mathrm{L}$

All counts

Tuberculosis (treatment of latent tuberculosis infection)

Coccidiodomycosis

Pneumocystis jirovecii

pneumonia

Histoplasmosis

$\leq 150$

Talaromycosis

Toxoplasmosis

$<100$

Mycobacterium avium

$<50$
Indication

Regions

Recommended prophylaxis (first line)

Positive tuberculin skin test or interferon- $\gamma$ release assay result without evidence of active disease or previous treatment for active or latent tuberculosis

New positive result of IgM or IgG Coccidiodes spp. serologic testing (monitored annually if residence in endemic area)

All patients with CD4 count $<200$ cells/ $\mu \mathrm{L}$

- Residence in or travel to hyperendemic area

- High-risk activity (exploring caves, occupational exposure to bird or bat droppings) in endemic areas

Residence in or travel to endemic area

Positive result of IgG Toxoplasma gondii serologic testing

Not receiving fully suppressive antiretroviral therapy after active Mycobacterium avium complex ruled out
All regions

soniazid, $300 \mathrm{mg} / \mathrm{d}$ orally, + pyridoxine, $25-50 \mathrm{mg} / \mathrm{d}$ orally

Southern California, Arizona; southern Utah, Nevada, New Mexico; western Texas

All regions

- French Guiana, some other regions of South America

- Mississippi, Ohio, St. Lawrence River valleys; Caribbean, southern Mexico

- Some regions of Central America, Africa, Asia

Northern Thailand, Vietnam, southern China

All regions

All regions

Fluconazole, $400 \mathrm{mg} / \mathrm{d}$ orally
Trimethroprimsulfamethoxazole, 1 tablet single strength orally daily Itraconazole, $200 \mathrm{mg} / \mathrm{d}$ orally

traconazole, $200 \mathrm{mg} / \mathrm{d}$ orally

Trimethroprimsulfamethoxazole, 1 tablet double strength orally daily

Azithromycin, 1200 mg orally once weekly

\section{References}

1. Fungal infections. Talaromyces marneffei infection. Cheshire (UK): Leading International Fungal Infection (LIFE). Available: www.life-worldwide.org/ fungal-diseases/talaromyces-marneffei-infection (accessed 2019 Nov. 21).

2. Limper AH, Adenis A, Le T, et al. Fungal infections in HIV/AIDS. Lancet Infect Dis 2017;17:e334-43.

3. Chan JF, Lau SK, Yuen KY, et al. Talaromyces (Penicillium) marneffei infection in non-HIV-infected patients. Emerg Microbes Infect 2016;5:e19.

4. Chariyalertsak S, Sirisanthana T, Supparatpinyo K, et al. Case-control study of risk factors for Penicillium marneffei infection in human immunodeficiency virus-infected patients in northern Thailand. Clin Infect Dis 1997;24:1080-6.

5. Chastain DB, Henao-Martínez AF, Franco-Paredes C. Opportunistic invasive mycoses in AIDS: cryptococcosis, histoplasmosis, coccidiodomycosis, and talaromycosis. Curr Infect Dis Rep 2017;19:36.

6. Larsson M, Nguyen LH, Wertheim HF, et al. Clinical characteristics and outcome of Penicillium marneffei infection among HIV-infected patients in northern Vietnam. AIDS Res Ther 2012;9:24.

7. Jiang J, Meng S, Huang S, et al. Effects of Talaromyces marneffei infection on mortality of HIV/AIDS patients in southern China: a retrospective cohort study. Clin Microbiol Infect 2019;25:233-41.

8. Ning C, Lai J, Wei W, et al. Accuracy of rapid diagnosis of Talaromyces marneffei: a systematic review and meta-analysis. PLoS One 2018;13:e0195569.

9. Kauffman CA. Central nervous system infection with other endemic mycoses: rare manifestation of blastomycosis, paracoccidiodomycosis, talaromycosis and sporotrichosis. J Fungi (Basel) 2019;5:64.

10. Panel on Opportunistic Infections in Adults and Adolescents with HIV. Guidelines for the prevention and treatment of opportunistic infections in adults and adolescents with HIV: recommendations from the Centers for Disease Control and Prevention, the National Institutes of Health, and the HIV Medicine Associa-

tion of the Infectious Diseases Society of America. Rockville (MD): AIDSinfo. Available: http://aidsinfo.nih.gov/contentfiles/lvguidelines/adult_oi.pdf (accessed 2019 Aug. 27).

11. Castro-Lainez MT, Sierra-Hoffman M, LLompart-Zeno J, et al. Talaromyces marneffei infection in a non-HIV non-endemic population. IDCases 2018;12:21-4.

\section{Competing interests: None declared.}

This article has been peer reviewed.

The authors have obtained patient consent.

Affiliations: Department of Medicine (Waters) and Division of Infectious Diseases (Beliavsky, Gough), Department of Medicine, University of Toronto; Division of Infectious Diseases (Gough), St. Michael's Hospital, Toronto, Ont.

Contributors: Mara Waters and Alina Beliavsky drafted the manuscript. All of the authors revised the manuscript critically for important intellectual content, approved the final version to be published and agreed to be accountable for all aspects of the work.

Acknowledgements: The authors thank Dr. Manal Tadros, Dr. Larissa Matukas, Dr. Ramzi Fattouh, the St. Michael's Microbiology Laboratory, Dr. Julianne Kus and the Public Health Ontario laboratory for their contributions to the microbiologic testing in this case.

Correspondence to: Mara Waters, mara.waters@mail.utoronto.ca 